\title{
Study on the Application of New Materials in Chemical Protective Clothing
}

\author{
Dai Xuezhi ${ }^{1, a}, \quad$ Xu Jingkai ${ }^{2}$ \\ ${ }^{1}$ Institute of NBC Defense, Chinese PLA Army, Beijing, China \\ 232102 Army, Shandong, China
}

\begin{abstract}
It is necessary to protect the safety of people in certain occupations where they might be exposed to hazardous chemicals. This review provided an overview of chemical protective clothing, along with its necessity during industrial and military operations as well as in response to acts of terror. Moreover, chemical protection or barrier suits are illustrated and explained including their types, selection processes based on chemical hazards, working environments, and various materials available for the fabrication of effective barrier clothing. Additionally, the current research gaps were elucidated in this review, so as the challenges facing recently developed chemical protective clothing, and relevant research are compiled onto a single platform. Moreover, the future development trend of chemical protective equipment based on new materials and technologies was also explored and analyzed.
\end{abstract}

\section{Introduction}

Protective textile is a kind of material used to weave protective clothing or other protective products. Among all kinds of hazards, chemicals and chemical agents are considered the most dangerous ${ }^{[1-4]}$. Chemical protective clothing is a kind of chemical protective equipment widely used by military personnel, law enforcement personnel and civilians in wartime and peacetime, including chemical warfare, industrial chemical production, chemical leakage, riots and anti-terrorism activities. Chemical protective clothing includes respirators, masks, coats, boots, gloves, etc. Meanwhile, researches are focusing on the development of a system providing people with more effective protection and preventing contact with toxic chemicals. For example, protective gloves, typically with five fingers, are made of various materials and designed with different thicknesses and styles for safe handling of hazardous chemicals like corrosive acids and alkalis ${ }^{[5]}$. Various polymer materials, such as butyl rubber, nitrile rubber, chloroprene rubber and natural rubber, are usually used in the preparation of such protective gloves based on the properties of targeted chemicals. In addition, chemical and biological masks are used to protect users from chemical and biological pollution in emergencies when there are highly toxic substances in the air. This mask forms a sealed protective surface on fragile soft tissues of the face, such as nose, mouth and eyes ${ }^{[6]}$.

Chemical protective clothing boasts waterproof and oil-proof properties via halogenated polymers in the form of lamination or coating. These coated fabrics have low surface energy, thus resisting the infiltration of water and organic liquid by lotus effect ${ }^{[7]}$. Chemical protective clothing with good air permeability has a microporous membrane that can block liquid chemicals while allowing air and water vapor to penetrate. Chemical protective clothing made of activated carbon-based textile is also widely used for military and civil purposes ${ }^{[5-6]}$. The combination of textile materials and activated carbon provides ideal thermal conductivity and transfers heat well, and the activated carbon materials can effectively protect the skin from harmful chemicals via adsorption and activation properties.

It is necessary to study the definition, protective capabilities, compatibility and integration of different protective materials that serve as the major component of chemical protective clothing in the design of chemical protection against harmful chemicals and microorganisms ${ }^{[8]}$. In addition, the release of body moisture should be minimized and air circulation must be maintained to ensure permeability and air permeability of the material. How to balance between the protection of human body and user experience remains the major challenge confronting us. Therefore, this paper started with the selection of chemical protective materials, and then explored in detail their performance against dangerous chemicals or chemical agents by combining a variety of new materials and technologies.

\section{Technical analysis of new materials for chemical protective clothing}

The new materials provide protective clothing with novel characteristics such as detecting harmful chemicals on the fabric surface, eliminating toxic chemicals in contact with the fabric, and selectively blocking harmful chemicals,

a Corresponding author: 297896810@qq.com 
which is considered as a key in future development. New materials that enhance the performance of chemical protective clothing mainly include active nanofiber and nanoparticles, conductive polymers and catalytic materials based on biological enzymes. The new generation of chemical protective clothing developed with these new materials and methods could be applied in specific field such as isolation protection and detection $[9,10]$.

\subsection{Nano-textile}

Nanotechnology also plays a unique role in textile materials, and is developing vigorously in an unprecedented way. Therefore, with the growing popularity of nanotechnology, researchers around the world are also contribute to the development of advanced materials for chemical protection ${ }^{[11]}$.

The latest studies on nanomaterials includes nanoparticles, nanorods, nanotubes, nanowires and nanocomposites. Metal nanoparticles, such as $\mathrm{Ag}, \mathrm{Au}, \mathrm{Pd}$, $\mathrm{Cu}, \mathrm{Ni}$, and metal oxide nanoparticles, such as $\mathrm{MgO}, \mathrm{CaO}$, $\mathrm{ZnO}, \mathrm{TiO}_{2}, \mathrm{Al}_{2} \mathrm{O}_{3}, \mathrm{MnO}_{2}, \mathrm{Fe}_{2} \mathrm{O}_{3}$, etc., have been reported in many scientific literatures because of their excellent catalytic properties and outstanding performances ${ }^{[12]}$. As effective catalysts against chemical agents, they serve as reliable chemical protective materials by adsorbing and decomposing toxic substances simultaneously.

Nanoparticles prepared via aerogel are widely used in chemical protective clothing because of their relatively large surface $\left(>400 \mathrm{~m}^{2} / \mathrm{g}\right)$ and porosity $(>85 \%)$. Meanwhile, those dangerous chemical agents are turned into harmless substances through irreversible decomposition.

Metal oxide nanoparticles also endow textile fibers with functional characteristics through surface modification. Protective textile materials interact with metal oxide nanoparticles to decompose malodorous molecules, harmful bacteria and other organic substances and destroy toxic chemicals through photocatalysis. However, the modification on the surface of textile fiber by metal oxides also has limitations. In the fabric surface coating, the agglomeration of particles is a challenge as the particles will not stay on the fabric surface permanently. The fabric treated with nanoparticles will not only deteriorate the appearance of the fabric, but also reduce the flexibility and wear resistance of the fabric. Therefore, it is necessary to find an effective method to stabilize dispersed particles ${ }^{[13]}$.

\subsection{Nanofibers}

Nanofiber is a fine material with nanometer diameter, which is usually produced by electrospinning ${ }^{[14-17]}$. The potential applications of nanofiber materials in protective clothing have been confirmed by multiple modern studies. Light-weight electrospun nanofiber clothing materials show greater air permeability and retention of fluid and aerosol particles.

In the field of chemical protective clothing, electrospun nanofiber materials have a wide range of potential applications, including protections against chemical agents, liquid infiltration, and agricultural chemicals. Electrospun nanofiber web can be used as a membrane against liquid permeation in chemical protective clothing. Studies show that a thin polyurethane nanofiber web can significantly improve the performance of separating liquids with special physical and chemical properties. Schreud, Gibson and others explored the protective layer of fine submicron fibers produced by electrospinning to prevent penetration of chemical agents in aerosol form. They observed that electrospun nanofiber materials with different polymers can provide solid aerosol particle protection while there were no drastic changes in the water vapor transmission rate of the module. Compared with traditional coating, multilayer fabrics based on electrospun materials have better air and moisture permeability. Other functional compounds, such as cyclodextrin (-CD), iodobenzoic acid (IBA), polyoxometalates, peroxides, oximes and chloramines, can be added to electrospun fiber materials to enhance their detoxification performance. The polymer nanofibers with active compounds have a higher hydrolysis rate for organic compounds, as shown in Table 1.

Table 1. Relative Hydrolysis Rate of Organophosphorus by Different Nanofiber Membranes

\begin{tabular}{lc} 
Polyvinyl Chloride Nanofibers & Relative Hydrolysis Rate \\
\hline Activated Charcoal & 1.0 \\
$\beta$-cyclodextrin & 1.75 \\
Iodinyl Benzoic Acid & 2.5 \\
Mixture of $\beta$-cyclodextrin and & 5.5 \\
Iodoxybenzoic Acid & \\
\hline
\end{tabular}

With zinc titanate $\left(\mathrm{ZnO}-\mathrm{TiO}_{2}\right)$ as the raw material, a new type of ceramic nanofiber was developed via sol-gel, and was used as protective clothing and masks against harmful chemicals. The synthesized nanofibers, with a higher porosity and larger surface, can decompose nerve and mustard gas simulants into nontoxic products through adsorption and detoxification.

\subsection{Conductive polymers}

Conductive polymer is a novel organic material with conductivity leading to an exciting new field of material science. It features good electrical properties, active redox potential, stability and processability, fast reaction kinetics rate and reversible absorption.

Conductive nanofibers are usually prepared by electrospinning, and the development of electrospun nanofiber includes various polymers, such as polyaniline (PANI), polypyrrole (PPY) and poly $(3,4-$ ethyl dioxythiophene) (PEDOT) ${ }^{[14,19]}$. The electronic properties of these polymers are characterized by their reactions to various gases, including organic vapors, liquid acids and bases. Among the conductive polymers, PANI provides an ideal solution because of its good 
conductivity, low cost, transparency, and stability in terms of matrix mechanics, allowing effective adjustment through doping. The oxidation form of PANI can be transformed from insulating jadeite alkali form $\left(\sigma<10^{-10}\right.$ $\mathrm{S} / \mathrm{cm})$ to conductive jadeite salt form $(\sigma>1 \mathrm{~S} / \mathrm{cm})$ by acid or alkali doping and undoping, as shown in Figure 1. This fluctuation of conductivity can be used to develop PANI sensors for detecting toxic industrial chemicals.

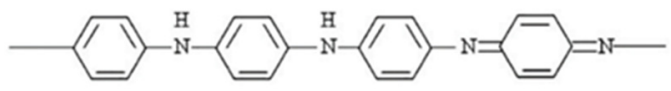

Emeraldine base (insulating state)

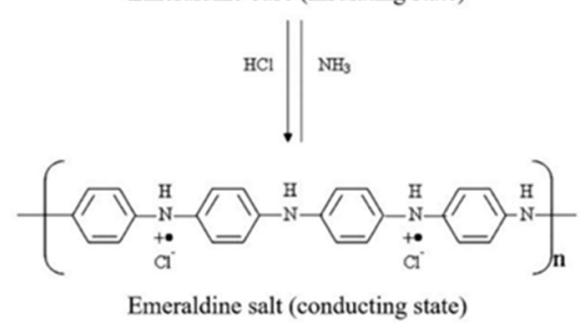

Figure 1. Diagram of Switching Mechanism between Carborundum Alkali (Insulating Type) and Carborundum Salt (Conductive Type) Using Hydrochloric Acid and Ammonia Water Respectively

The presence of chemical pollutants would cause changes of the optical properties of fibers in fabrics when there are fluctuations in the oxidation state of conductive polymers. Conductive polymers, such as PANI and PPY, show reversible changes when exposed to chemical vapor, leading to changes of the optical properties of fabrics. The change of optical properties caused by chemical interaction is related to refractive index and optical absorption spectrum of polymer films.

There are many gas sensors using conductive polymers as sensing elements, featuring great flexibility and the ability to detect deadly chemical gases by changing light absorption. Studies found drastic changes in the UV-vis absorbance band of conductive PPY films (a decrease of $40 \%$ ) after being exposed to DMMP. This is probably the result of electronic interactions between DMMP and PPY that increase the number of free-moving hole charge carriers, causing changes to the volume, conductivity and light absorption properties of these carriers. In addition, due to interactions between hydrazine $\left(\mathrm{N}_{2} \mathrm{H}_{4}\right)$ and PPY film, the refractive index decreased from 1.82 to 1.71 before and after contact with hydrazine.

\subsection{Enzyme-based Chemical Protective Clothing}

Enzymes are considered as an efficient biocatalyst, which acts as catalysts for specific biochemical reactions. Many enzymes can degrade various organic phosphorus compounds. By adding enzymes and polymers, various self-cleaning surface materials can be developed for the design of chemical protective clothing.

Among various enzymes, organophosphorus hydrolase (OPH) is known for its good hydrolytic activity against OP pesticides, including paraoxon, parathion, coumaphos and diazinon, as well as nerve agents such as sarin, soman and VX. However, the hydrolytic activity of $\mathrm{OPH}$ to chemical agent VX is less than $0.1 \%$, much lower than its activity to parathion and paraoxon. The detoxification of organophosphorus neurotoxins by $\mathrm{OPH}$ mainly depends on hydrolysis between central phosphorus and the leaving group through various phosphate bonds (P-O, P-F, P-CN, P-S).

Paraoxonase-1 (PON1) is another type of enzyme considered as an efficient catalytic biological scavenger because it can hydrolyze many OPs, including pesticides and nerve agents. This enzyme exists in all mammals. Most of the enzymes are synthesized in liver, and some secreted into plasma to combine with high density lipoprotein (HDLs). PON1 is a calcium-dependent hydrolase and the most popular topic for researchers among calcium-dependent hydrolases. PON1 is active in hydrolyzing phosphotriester pesticides such as paraoxone, sarin or soman. In terms of its structure, PON1 is an active site where two calcium ions are folded through the hexalobal $\beta$-helix cycle to catalyze $\mathrm{Ca}^{2+}$ in its central tunnel. The active site of PON1 reveals a strong network and versatility, allowing the combination of different substrates to catalyze chemical reactions. This enzyme and its mutant can be used as potential coating of chemical protective paint.

\section{Conclusions}

The combination of advanced fabric materials and novel technologies offers the chemical clothing unique functions, including the ability to detect, inactivate toxic chemicals and selectively block dangerous chemicals. By detecting, capturing and purifying toxic particles, liquids and vapors, the self-detoxification innovative fabric could deactivate nanoparticles and nanofibers by chemical methods, providing comprehensive chemical protection. In addition, enzyme-based high polymer materials will be used as high-efficiency biocatalysts to develop advanced self-cleaning copolymers, which will provide early warning of environmental hazards for users. With the emergence of the above novel technologies, the traditional product will be replaced by new chemical protective clothing.

\section{References}

1. Zhang, Y., Miao, J., Han, L.X. (2019) Optimizing Clothing Supporting High Temperature operation [J]. Journal of Nantong Vocational University. 33(03):59-62.

2. Liu, L., Zhang, S.H., Cai, P.N., Li, S.X., Chen, Z.T. (2019) Development of Flame Retardant Viscose Wool Blend for Preventing Molten Metal Splash [J]. Cotton Textile Technology. 47(11):29-32.

3. Zhang, T.T., Ren, Z.B., Xu, L.J., Dong, B.Y., Zhang, H. (2019) Problems in Testing of Thermal Protection Performance of Flame Retardant Protective Clothing [J]. Wool Textile Science and Technology. 47(11):69-71. 
4. Han, Y. (2019) Study on Flame Retardant Chemical Protective Clothing [J]. Labor Protection. (11):14-15.

5. Hu, Z.T., Zheng, X.H., Feng, M.M., Wang, Y.J., Li, L., Ding, S.T. (2019) Influence of Air Layer on Thermal Resistance and Moisture Resistance of Airpermeable Protective Clothing [J]. Journal of Textile Research. 40(11):145-150.

6. Sadeghi M., Yekta S., Ghaedi H. (2016) Decontamination of chemical warfare sulfur mustard agent simulant by $\mathrm{ZnO}$ nanoparticles [J]. Int Nano Lett. 6:161-171.

7. Paukku Y., Michalkova A., Leszczynski J. (2008) Adsorption of dimethyl methylphosphonate and trimethyl phosphate on calcium oxide: An ab initio study [J]. Struct Chem. 19: 307-320.

8. Gershonov E., Columbus I., Zafrani Y. (2008) Facile hydrolysis-based chemical destruction of the warfare agents VX, GB, and HD by alumina-supported fluoride reagents [J]. J. Org. Chem. 74: 329-338.

9. Zhu, L.J., Fu, J., Chun, H. (2019) Design of Safe and Comfortable Protective Clothing [J]. Labor Protection, (11):101.

10. Yao, W.C., Fu, Y.H., Jiang, J.X. (2019) Mathematical Model of Special Clothing Supporting High Temperature Operation [J]. Electronic Testing, (Z1):63-65.

11. Sayago I., Matatagui D., Ferna' ndez M.J. (2016) Graphene oxide as sensitive layer in Love-wave surface acoustic wave sensors for the detection of chemical warfare agent simulants [J]. Talanta. 148: 393-400.

12. Shen, Y., Yue, M.G., Bao, W.L. (2019) Study on the Optimal Design of Special Protective Clothing for High Temperature Operation Based on Multi-layer Plane Wall Heat Conduction Model [J]. Chemical Management. 2:104-105.

13. Liu, J., Sun, Y.R. (2018) Development and Prospect of Laboratory Biosafety Protective Equipment in China [J]. China Public Health. 34(12):1700-1704.

14. Eigler, S., Dotzer, C., Hof, F. (2013) Sulfur species in graphene oxide [J]. Chem Eur J. 19:9490-9496.

15. Zhang, X.Y., Tian, L., Wang, G.H., Xu Z.G. (2012) Solution to Heat Load Caused by Heavy Chemical Protective Clothing [J]. Fire Science and Technology. 31(01):5-8.

16. Li, G.M., Wang J., Yang, M. (2011) Chemical Protective Clothing[J]. Life and Disaster. 06:1.

17. He, Z.Y. (2011) Discussion on Responses to Chemical Accidents by Fire Brigade Using Thiourea Pyrolysis [J]. Fire Science and Technology. 30(03):243-245.

18. Zhang, K.Z. (2011) Development of Polyphenylene Sulfide (PPS) for Outer Fabric of Chemical Protective Clothing [D]. Shanghai: Donghua University.

19. Wang, Y., Liu, M., Wang, X.D. (2010) Classification and Selection of Chemical Protective
Clothing [J]. Personal Protective Equipment in China. 01:32-37. 